\title{
PAPER \\ An Iterative MIMO Receiver Employing Virtual Channels with a Turbo Decoder for OFDM Wireless Systems
}

\author{
Akihito TAYA $^{\dagger}$, Member, Satoshi DENNO $^{\dagger \dagger a)}$, Koji YAMAMOTO $^{\dagger}$, Senior Members, \\ Masahiro MORIKURA ${ }^{\dagger}$, Fellow, Daisuke UMEHARA ${ }^{\dagger \dagger}$, Hidekazu MURATA $^{\dagger}$, Senior Members, \\ and Susumu YOSHIDA ${ }^{\dagger}$, Fellow
}

\begin{abstract}
SUMMARY This paper proposes a novel iterative multiple-input multiple-output (MIMO) receiver for orthogonal frequency division multiplexing (OFDM) systems, named as an "iterative MIMO receiver employing virtual channels with a Turbo decoder." The proposed MIMO receiver comprises a MIMO detector with virtual channel detection and a Turbo decoder, between which signals are exchanged iteratively. This paper proposes a semi hard input soft output (SHISO) iterative decoding for the iterative MIMO receiver that achieves better performance than a soft input soft output (SISO) iterative decoding. Moreover, this paper proposes a new criterion for the MIMO detector to select the most likely virtual channel. The performance of the proposed receiver is verified in a $6 \times 2$ MIMO-OFDM system by computer simulation. The proposed receiver achieves better performance than the SISO MAP iterative receiver by $1.5 \mathrm{~dB}$ at the bit error rate (BER) of $10^{-4}$, by optimizing the number of the Turbo iteration per the SHISO iteration. Moreover, the proposed detection criterion enables the proposed receiver to achieve a gain of $3.0 \mathrm{~dB}$ at the BER of $10^{-5}$, compared with the SISO MAP iterative receiver with the Turbo decoder.

key words: multiple-input multiple-output, virtual channel, soft-input softoutput, log likelihood ratio, iterative scheme
\end{abstract}

\section{Introduction}

Since a lot of online applications are available even on mobile or wireless devices, high-speed wireless access networks are demanded for the applications. As a result, broad band wireless communication systems such as the long term evolution (LTE) and the IEEE 802.11ac have been developed where orthogonal frequency division multiplexing (OFDM) [1] is applied to combat with severe multipath fading, and multiple-input multiple-output (MIMO) [2], [3] is employed to increase the transmission speed.

In principle, transmission speed can be increased in MIMO systems by increasing spatially multiplexed streams. The MIMO spatial multiplexing has been intensively investigated for downlinks of wireless communications where signals are transmitted from base stations or access points to terminals, because higher speed data transmission is required in downlinks than in uplinks. As a result, many receiver configurations have been proposed, e.g., minimum mean square error (MMSE) filters, ordered successive de-

\footnotetext{
Manuscript received August 22, 2014.

Manuscript revised November 18, 2014.

${ }^{\dagger}$ The authors are with the Graduate School of Informatics, Kyoto University, Kyoto-shi, 606-8501 Japan.

${ }^{\dagger}$ The author is with the Graduate School of Natural Science and Technology, Okayama University, Okayama-shi, 700-8530 Japan.

${ }^{+\dagger}$ The author is with the Graduate School of Science and Technology, Kyoto Institute of Technology, Kyoto-shi, 606-8585 Japan.

a)E-mail: denno@cne.okayama-u.ac.jp
}

DOI: $10.1587 /$ transcom.E98.B.878 tectors (OSDs), eigenbeam-space division multiplexing (ESDM), and the maximum likelihood detection (MLD) with QR decomposition and M-algorithm (QRM-MLD) [4]. Basically, if these linear MIMO detectors are applied in MIMO systems, the maximum number of the spatially multiplexed streams is equal to the number of degrees of freedom that is $\min \left(N_{\mathrm{T}}, N_{\mathrm{R}}\right)$ where $N_{\mathrm{T}}$ and $N_{\mathrm{R}}$ denote the number of transmit antennas and that of receive antennas, and $\min ()$ is a function of outputting the smallest value in the input values. When these schemes are used in downlinks, the transmission speed is limited by the number of receive antennas, because terminals do not have enough room to install many antennas while base stations or access points do. In other words, base station antennas are not fully exploited to increase the transmission speed for a user. In order to increase the transmission speed for a user, some receivers have been proposed that can be applied in MIMO systems where the number of the spatially multiplexed streams is more than that of receive antennas [5], [6]. These receivers assume that the number of the streams is by about one more than that of the receive antennas. Those receivers are classified into linear detectors. In contrast with linear detectors, non-linear detectors achieve superior performance in MIMO systems where the number of spatially multiplexed streams is more than that of the receive antennas. For instance, the detector with "virtual channels" achieves superior performance with reasonable complexity even when the number of the streams is twice as many as that of the receive antennas [7]-[9]. Moreover, the SISO iterative receiver composed of the MIMO detector with virtual channels and the convolutional decoder has been proposed for further performance improvement [9].

On the other hand, Turbo codes [10] have been considered to combine with MIMO detectors [11]-[14], because Turbo codes are powerful to improve transmission performance. However, even if Turbo decoders are applied to the SISO iterative receiver with the MIMO detector with the virtual channel detection, the performance is not improved, which is shown in this paper.

This paper proposes a novel iterative receiver, called as a semi hard input soft output (SHISO) iterative receiver, that fully exploits the potential gain of Turbo decoders to achieve superior performance even when the number of the streams is more than twice as many as that of the receive antennas. The SHISO iterative receiver outperforms a SISO iterative receiver, which is shown in the following section. 


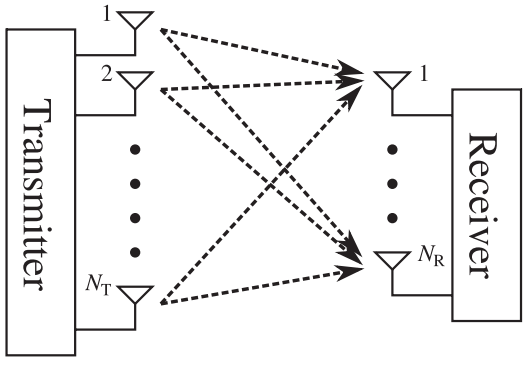

Fig. 1 System model.

Basically, the proposed receiver comprises a MIMO detector with virtual channel detection and the Turbo decoder, between which signals are exchanged iteratively. The ordered successive detector (OSD) is used to detect the signal in each virtual channel of the MIMO detector ${ }^{\dagger}$. In the proposed receiver, the extrinsic information is fed back from the Turbo decoder to the MIMO detector, and the MIMO detector estimates the phase on the maximum a posteriori probability (MAP) estimation with the extrinsic information. However, the hard decision signal of the phase is provided to the Turbo decoder, whilst the ordered successive detector output signals are fed to the decoder. This SHISO iteration has not been proposed before and is a key idea to improve the performance.

Moreover, we propose a new criterion, "Extended MAP estimation", for the proposed receiver to select the most likely virtual channel for furthermore improving the transmission performance with little additional complexity.

The rest of this paper is structured as follows. Section 2 introduces a model of the MIMO system. Section 3 describes a configuration of our proposed receivers with virtual channels, and Sect. 4 shows the performance of the proposed receivers, obtained by computer simulations. Finally, Sect. 5 presents concluding remarks.

\section{System Model}

We consider a downlink with $N_{\mathrm{T}}$ antennas at a transmitter and $N_{\mathrm{R}}$ antennas at a receiver. At the transmitter, data bits are encoded using a Turbo code, interleaved, and modulated into Quaternary phase-shift keying (QPSK) signals. The QPSK signals are then modulated on the orthogonal subcarriers in the system with the OFDM. Let $n$ denote a time index, the output signal vector $\boldsymbol{d}_{n} \in \mathbb{C}^{N_{\mathrm{T}} \times 1}$ is written as,

$$
\boldsymbol{d}_{n}=\frac{1}{\sqrt{N}} \sum_{k=0}^{N-1} \hat{\mathbf{D}}_{k} \mathrm{e}^{\mathrm{j} 2 \pi \frac{n k}{N}}
$$

where $k, \hat{\mathbf{D}}_{k} \in \mathbb{C}^{N_{\mathrm{T}} \times 1}$ and $N$ denote a subcarrier index, a

${ }^{\dagger}$ The OSD is one of serial interference cancellers, which is applied in the proposed iterative receiver. On the other hand, interference is cancelled by using replicas of the interference signals that are generated from a priori information fed back from the decoder in Turbo equalizers, which are well known as iterative receivers. Hence, the proposed receiver is completely different from Turbo equalizers [15]-[17]. modulation signal vector, and the number of the subcarriers. The output signal vector is emitted from the transmit antennas, and is travelling multipath fading channels. Then, the vector is received at the receive antennas, which is expressed as follows.

$$
\boldsymbol{y}_{n}=\sum_{t=0}^{T-1} \boldsymbol{h}_{t} \boldsymbol{d}_{n-t}+\boldsymbol{n}_{n},
$$

where $\boldsymbol{y}_{n} \in \mathbb{C}^{N_{\mathrm{R}} \times 1}, \boldsymbol{h}_{t} \in \mathbb{C}^{N_{\mathrm{R}} \times N_{\mathrm{T}}}, \boldsymbol{n}_{n} \in \mathbb{C}^{N_{\mathrm{R}}}$ denote a received signal vector, a channel matrix of $t$ th path, the additive white Gaussian noise (AWGN) vector. In addition, $T$ represents the maximum delay of the channel that is assumed not to exceed the cyclic prefix length. The receiver converts the received signal vector in the time domain into the frequency domain with the fast Fourier transform (FFT). The frequency domain signal vector in the $k$ th subcarrier, $\hat{\mathbf{Y}}_{k} \in \mathbb{C}^{N_{\mathrm{R}} \times 1}$, is expressed as

$$
\begin{aligned}
\hat{\mathbf{Y}}_{k} & =\frac{1}{\sqrt{N}} \sum_{n=0}^{N-1} \boldsymbol{y}_{n} \mathrm{e}^{-\mathrm{j} 2 \pi \frac{n k}{N}} \\
& =\hat{\mathbf{H}}_{k} \hat{\mathbf{D}}_{k}+\hat{\mathbf{N}}_{k},
\end{aligned}
$$

where $\hat{\mathbf{H}}_{k}=\sum_{t=0}^{T-1} \boldsymbol{h}_{t} \mathrm{e}^{-\mathrm{j} 2 \pi \frac{k t}{N}} \in \mathbb{C}^{N_{\mathrm{R}} \times N_{\mathrm{T}}}$ and $\hat{\mathbf{N}}_{k}=$ $\frac{1}{\sqrt{N}} \sum_{n=0}^{N-1} \boldsymbol{n}_{n} \mathrm{e}^{-\mathrm{j} 2 \pi \pi^{n k} N} \in \mathbb{C}^{N_{\mathrm{R}} \times 1}$ represent a channel matrix and the AWGN at the $k$ th subcarrier. In principle, complex signal vectors and channel matrices can be transformed into real vectors and matrices, respectively [7]-[9]. Let $\mathbf{Y}_{k} \in \mathbb{R}^{2 N_{\mathrm{R}}}, \mathbf{D}_{k} \in \mathbb{R}^{2 N_{\mathrm{T}}}, \mathbf{N}_{k} \in \mathbb{R}^{2 N_{\mathrm{R}}}$, and $\mathbf{H}_{k} \in \mathbb{R}^{2 N_{\mathrm{R}} \times 2 N_{\mathrm{T}}}$ represent the received signal vector, the data signal vector, the AWGN vector, and the channel matrix expressed in real values, respectively. (3) can be rewritten as

$$
\mathbf{Y}_{k}=\mathbf{H}_{k} \mathbf{D}_{k}+\mathbf{N}_{k} .
$$

Because the same signal processing is performed in every subcarrier, the subcarrier index $k$ is omitted for simplicity in the rest of this paper, which does not confuse readers. The $l$ th entry of $\mathbf{D}, d_{l}$, is decomposed into a real signal $s_{l}$ and a phase $\theta_{l}$ as $d_{l}=s_{l} \mathrm{e}^{\mathrm{j} \theta_{l}}$, where $s_{l}= \pm \sqrt{2}$ and $\theta_{l}= \pm \pi / 4$. When the decomposition of a scalar is extended to a vector, D is rewritten as follows [7].

$$
\begin{aligned}
& \mathbf{D}=\boldsymbol{\Omega}(\varphi) \mathbf{S}(\varphi), \\
& \boldsymbol{\Omega}(\varphi)=\left[\begin{array}{l}
\operatorname{diag}\left[\cos \theta_{1}, \cdots, \cos \theta_{N_{\mathrm{T}}}\right. \\
\operatorname{diag}\left[\sin \theta_{1}, \cdots, \sin \theta_{N_{\mathrm{T}}}\right]
\end{array}\right], \\
& \mathbf{S}(\varphi)=\left[\begin{array}{lll}
s_{1}(\varphi) & \cdots & s_{N_{\mathrm{T}}}(\varphi)
\end{array}\right]^{\mathrm{T}},
\end{aligned}
$$

where diag $[\mathbf{V}]$ represents a diagonal matrix with entries of a vector $\mathbf{V}$ in the diagonal positions and superscript $\mathrm{T}$ represents transpose of a vector. We call $\boldsymbol{\Omega}(\varphi) \in \mathbb{R}^{2 N_{\mathrm{T}} \times N_{\mathrm{T}}}$ a rotation matrix, $\mathbf{S}(\varphi) \in \mathbb{R}^{N_{\mathrm{T}}}$ a real signal vector. We refer $\varphi$ as a "phase pattern," which is defined as

$$
\varphi=\sum_{l=1}^{N_{\mathrm{T}}}\left(\frac{\theta_{l}}{\pi / 2}+\frac{1}{2}\right) 2^{l-1} .
$$




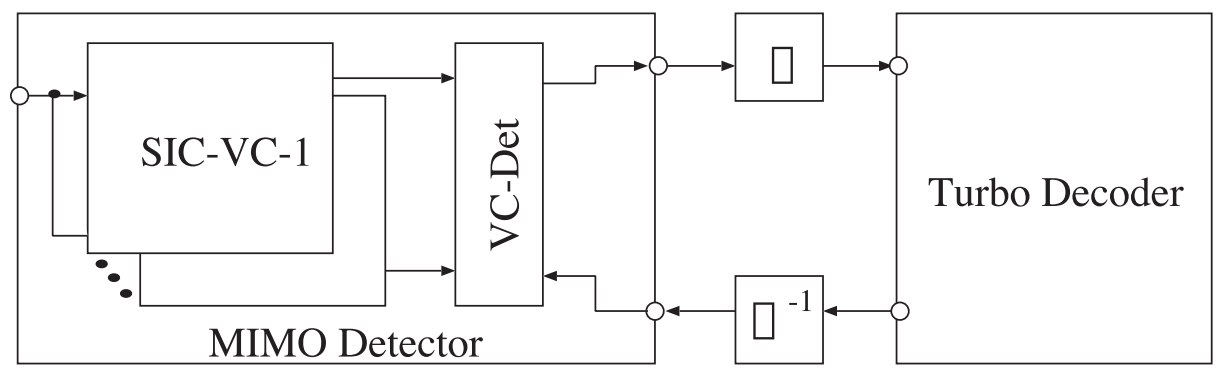

Fig. 2 Basic configuration of the receiver.

The phase pattern $\varphi$ ranges from 0 to $2^{N_{\mathrm{T}}}-1$. From (5), we can rewrite (4) as

$$
\begin{aligned}
& \mathbf{Y}=\boldsymbol{\Phi}(\varphi) \mathbf{S}(\varphi)+\mathbf{N}, \\
& \boldsymbol{\Phi}(\varphi)=\mathbf{H} \boldsymbol{\Omega}(\varphi) .
\end{aligned}
$$

As is expressed in (9), the channel model can be regarded that the signal vector $\mathbf{S}(\varphi)$ is transmitted in the channel $\boldsymbol{\Phi}(\varphi) \in \mathbb{R}^{2 N_{\mathrm{R}} \times N_{\mathrm{T}}}$, which is called "a virtual channel". While the size of the channel matrix $\mathbf{H}_{k}$ is $2 N_{\mathrm{R}} \times 2 N_{\mathrm{T}}$, that of the virtual channel $\boldsymbol{\Phi}(\varphi)$ is $2 N_{\mathrm{R}} \times N_{\mathrm{T}}$. In a word, the number of the columns of the virtual channel $\boldsymbol{\Phi}(\varphi)$ is half of that of the channel matrix $\mathbf{H}_{k}$, which means that the number of the spatially multiplexed streams in the virtual channel looks half of that in the channel $\mathbf{H}_{k}$. If the number of the streams is reduced, the streams can be detected with higher performance by linear detectors, such as the OSD.

The OSD detects the real signal vector in all the virtual channels, $\boldsymbol{\Phi}(\bar{\varphi}) \bar{\varphi}=0, \cdots, 2^{N_{\mathrm{T}}}-1$, where $\bar{\varphi}$ is a tentative phase pattern. In the OSD, basically, the transmission signal is detected with an MMSE filter, and the interference associated with the previously detected signals is canceled from the received signal vector. This step is iterated by $N_{\mathrm{T}}$ times in the OSD. The lth-step MMSE filter $\mathbf{W}^{(l)}(\bar{\varphi})$ is defined as

$$
\mathbf{W}^{(l)}(\bar{\varphi})=\left(\boldsymbol{\Phi}^{(l)}(\bar{\varphi})\left[\boldsymbol{\Phi}^{(l)}(\bar{\varphi})\right]^{\mathrm{T}}+\frac{\sigma_{\mathrm{n}}^{2}}{\sigma_{\mathrm{s}}^{2}} \boldsymbol{I}\right)^{-1} \boldsymbol{\Phi}^{(l)}(\bar{\varphi}),
$$

where $\sigma_{\mathrm{n}}^{2}, \sigma_{\mathrm{s}}^{2}$, and $\boldsymbol{I}$ represent the noise power, the signal power, and the unit matrix. In addition, $\boldsymbol{\Phi}^{(l)}(\bar{\varphi})$ denotes the virtual channel in the $l$ th step. Let $\boldsymbol{\Phi}^{(l)}(\varphi)$ be written as $\boldsymbol{\Phi}^{(l)}(\varphi)=\left[\boldsymbol{\phi}_{1}^{(l)}(\varphi), \cdots, \boldsymbol{\phi}_{N_{\mathrm{T}}}^{(l)}(\varphi)\right]$, the virtual channel in the $l$ th step is defined as $\boldsymbol{\Phi}^{(l)}(\bar{\varphi})=\left[0 \cdots \boldsymbol{\phi}_{l}^{(l)}(\bar{\varphi}), \cdots, \boldsymbol{\phi}_{N_{\mathrm{T}}}^{(l)}(\bar{\varphi})\right]$ where we assume that the signals are detected in the order of the antenna number for simplifying the notation. The $l$ th-stream signal is detected as

$$
s_{\mathrm{det}, l}(\bar{\varphi})=\left[\boldsymbol{w}_{l}(\bar{\varphi})\right]^{\mathrm{T}} \mathbf{Y},
$$

where $\boldsymbol{w}_{l}(\bar{\varphi})$ represents the $l$ th column of $\mathbf{W}^{(l)}(\bar{\varphi})$. The hard decision signal $\bar{s}_{\mathrm{det}, l}(\bar{\varphi})$ is defined as followings.

$$
\begin{aligned}
& \bar{s}_{\mathrm{det}, l}(\bar{\varphi})=\sqrt{2} \operatorname{sgn}\left[s_{\mathrm{det}, l}(\bar{\varphi})\right] \\
& \operatorname{sgn}[x]= \begin{cases}+1 & (x \geq 0) \\
-1 & (x<0)\end{cases}
\end{aligned}
$$

Using these hard decision signals, the interference cancellation is carried out as

$$
\mathbf{Y}^{(l+1)}=\mathbf{Y}^{(l)}-\boldsymbol{\phi}_{l}^{(l)}(\bar{\varphi}) \bar{s}_{\mathrm{det}, l}(\bar{\varphi}),
$$

where $\mathbf{Y}^{(1)}$ is set to $\mathbf{Y}$. The soft real signal vector $\mathbf{S}_{\mathrm{det}}(\bar{\varphi})$ and hard decision real signal vector $\overline{\mathbf{S}}_{\text {det }}(\bar{\varphi})$ are defined as

$$
\begin{aligned}
& \mathbf{S}_{\operatorname{det}}(\bar{\varphi})=\left[\begin{array}{lll}
s_{\operatorname{det}, 1}(\bar{\varphi}) & \cdots & s_{\operatorname{det}, N_{\mathrm{T}}}(\bar{\varphi})
\end{array}\right]^{\mathrm{T}}, \\
& \overline{\mathbf{S}}_{\operatorname{det}}(\bar{\varphi})=\left[\begin{array}{lll}
\bar{s}_{\operatorname{det}, 1}(\bar{\varphi}) & \cdots & \bar{s}_{\operatorname{det}, N_{\mathrm{T}}}(\bar{\varphi})
\end{array}\right]^{\mathrm{T}} .
\end{aligned}
$$

The most likelihood phase pattern is selected by a technique proposed in the following section. Figure 2 shows a basic configuration of the receiver. The real signals are detected by the OSD with the MMSE filter in each virtual channel, and the detected signals are provided to the virtual channel detector in the MIMO detector. The detector selects the most likely virtual channel based on the proposed technique described in the following section, and outputs the information associated with the signals to the Turbo decoder via the interleaver. The Turbo decoder estimates the log likelihood ratio (LLR) with the signal from the interleaver, and feeds back only the extrinsic information of the LLR to the MIMO detector.

\section{Proposed SHISO Iterative Receiver}

\subsection{MIMO Detector with Virtual Channels}

\subsubsection{LLR of Real Signals}

The LLR of the real signal $\lambda_{l}^{(s)}(\bar{\varphi})$ in the virtual channel $\boldsymbol{\Phi}(\bar{\varphi})$ can be defined as

$$
\lambda_{l}^{(\mathrm{s})}(\bar{\varphi})=\log \frac{\mathrm{P}\left(s_{l}(\bar{\varphi})=+\sqrt{2} \mid \mathbf{Y}\right)}{\mathrm{P}\left(s_{l}(\bar{\varphi})=-\sqrt{2} \mid \mathbf{Y}\right)},
$$

where $\mathrm{P}\left(s_{l}(\bar{\varphi})= \pm \sqrt{2} \mid \mathbf{Y}\right)$ represents an a posteriori probability of the real signal in the $l$ th-stream, $s_{l}(\bar{\varphi})$, when $\mathbf{Y}$ is received. The numerator of (18) can be approximately calculated with the Max-Log-MAP approximation as,

$$
\begin{aligned}
& \log \mathrm{P}\left(s_{l}(\bar{\varphi})=+\sqrt{2} \mid \mathbf{Y}\right) \\
& \simeq \log \mathrm{P}\left(s_{l}(\bar{\varphi})=+\sqrt{2}\right)-\log \mathrm{P}(\mathbf{Y})
\end{aligned}
$$




$$
+\max _{s_{l}(\bar{\varphi})=+\sqrt{2}}\left[\sum_{m \neq l} \log \mathrm{P}\left(s_{m}(\bar{\varphi})\right)-\frac{1}{2 \sigma_{\mathrm{n}}^{2}}|\mathbf{Y}-\boldsymbol{\Phi}(\bar{\varphi}) \mathbf{S}(\bar{\varphi})|^{2}\right],
$$

where $\mathrm{P}(\mathbf{S}(\bar{\varphi}))$ represents an a priori probability of $\mathbf{S}(\bar{\varphi})$. In addition, $\max _{\text {constraint }}\left[a_{m}\right]$ denotes the maximum value among $a_{m} m=0, \cdots$ under the indicated constraint. Because the exact calculation of (19) needs lots of complex calculations, the LLR of the real signal $\lambda_{l}^{(\mathrm{s})}(\bar{\varphi})$ is calculated more simply using the following hypothesis [9].

$$
|\mathbf{Y}-\boldsymbol{\Phi}(\bar{\varphi}) \mathbf{S}(\bar{\varphi})|^{2} \simeq \sum_{l}^{N_{\mathrm{T}}} \frac{\left(s_{\mathrm{det}, l}(\bar{\varphi})-s_{l}(\bar{\varphi})\right)^{2}}{\left|\boldsymbol{w}_{l}(\bar{\varphi})\right|^{2}}
$$

When the term in the left hand side of (20) is substituted for (19), the maximization with respect to $s_{m}(\bar{\varphi})$ can be performed independently. In a word, $s_{m}(\bar{\varphi})$ can be selected that satisfies $s_{m}(\bar{\varphi})=\max \left[\log \mathrm{P}\left(s_{m}(\bar{\varphi})\right)-\left(s_{\mathrm{det}, m}(\bar{\varphi})-s_{m}(\bar{\varphi})\right)^{2}\right]$. Therefore, the terms $s_{m}(\bar{\varphi}) m=1, \cdots, N_{\mathrm{T}}$ except $m=l$ become common between the numerator and the denominator in (18). Hence, the LLR of the real signals $\lambda_{l}^{(\mathrm{s})}(\bar{\varphi})$ can be approximately obtained as

$$
\begin{aligned}
\lambda_{l}^{(\mathrm{s})}(\bar{\varphi}) \simeq & \Lambda_{\mathrm{a}, l}^{(\mathrm{s})}(\bar{\varphi})-\frac{1}{2 \sigma_{\mathrm{n}}^{2}\left|\boldsymbol{w}_{l}(\bar{\varphi})\right|^{2}}\left\{\left|s_{\mathrm{det}, l}(\bar{\varphi})-\sqrt{2}\right|^{2}\right. \\
& \left.-\left|s_{\mathrm{det}, l}(\bar{\varphi})+\sqrt{2}\right|^{2}\right\} \\
= & \Lambda_{\mathrm{a}, l}^{(s)}(\bar{\varphi})+\frac{2 \sqrt{2} s_{\mathrm{det}, l}(\bar{\varphi})}{\sigma_{\mathrm{n}}^{2}\left|\boldsymbol{w}_{l}(\bar{\varphi})\right|^{2}} .
\end{aligned}
$$

where $\Lambda_{\mathrm{a}, l}^{(\mathrm{s})}(\bar{\varphi})$ represents a priori information of the real signal $s_{l}(\bar{\varphi})$, which is defined as

$$
\Lambda_{\mathrm{a}, l}^{(\mathrm{s})}(\bar{\varphi})=\log \frac{\mathrm{P}\left(s_{l}(\bar{\varphi})=+\sqrt{2}\right)}{\mathrm{P}\left(s_{l}(\bar{\varphi})=-\sqrt{2}\right)} .
$$

This a priori information is provided by the Turbo decoder following the detector.

By making a hard decision of $\lambda_{l}^{(\mathrm{s})}(\bar{\varphi})$, real signal vector $\tilde{\mathbf{S}}(\bar{\varphi})$ can be obtained in all the virtual channels.

$$
\begin{aligned}
\tilde{\mathbf{S}}(\bar{\varphi}) & =\left[\begin{array}{lll}
\tilde{s}_{1}(\bar{\varphi}) & \cdots & \tilde{s}_{N_{\mathrm{T}}}(\bar{\varphi})
\end{array}\right], \\
\tilde{s}_{l}(\bar{\varphi}) & =\sqrt{2} \operatorname{sgn}\left[\lambda_{l}^{(\mathrm{s})}(\bar{\varphi})\right] .
\end{aligned}
$$

The transmitted virtual channel is estimated with the estimated real signals by a technique proposed in the following section.

\subsubsection{LLR of Phase Pattern}

The proposed detector calculates the LLR of the phase pattern $\Lambda_{\bar{\varphi}}\left(\bar{\varphi}: \bar{\varphi}^{\prime}\right)$, in order to select the optimum phase pattern that maximizes an a posteriori probability. Here, $\bar{\varphi}^{\prime}$ is a phase pattern that differs from $\bar{\varphi}$. The LLR of the phase patterns is defined as

$$
\Lambda_{\bar{\varphi}}\left(\bar{\varphi}: \bar{\varphi}^{\prime}\right)=\log \frac{\mathrm{P}(\bar{\varphi} \mid \mathbf{Y})}{\mathrm{P}\left(\bar{\varphi}^{\prime} \mid \mathbf{Y}\right)}=\log \frac{\mathrm{P}(\mathbf{Y} \mid \bar{\varphi}) \mathrm{P}(\bar{\varphi})}{\mathrm{P}\left(\mathbf{Y} \mid \bar{\varphi}^{\prime}\right) \mathrm{P}\left(\bar{\varphi}^{\prime}\right)},
$$

where $\mathrm{P}(\bar{\varphi} \mid \mathbf{Y}), \mathrm{P}(\mathbf{Y} \mid \bar{\varphi})$, and $\mathrm{P}(\bar{\varphi})$ represent an a posteriori probability of the phase pattern, a conditional probability when a signal vector with a phase pattern $\bar{\varphi}$ is transmitted, an a priori probability of the virtual channel with the phase pattern $\bar{\varphi} \cdot P(\bar{\varphi})$ can be expressed as

$$
\mathrm{P}(\bar{\varphi})=\prod_{l=1}^{N_{\mathrm{T}}} \mathrm{P}\left(\theta_{l}\right),
$$

where $\mathrm{P}\left(\theta_{l}\right)$ represents a priori probability of the phase $\theta_{l}$ of the transmission signal from the $l$ th antenna. Using (26), (25) can be rewritten as

$$
\begin{aligned}
\Lambda_{\bar{\varphi}}\left(\bar{\varphi}: \bar{\varphi}^{\prime}\right)= & \sum_{l=1}^{N_{\mathrm{T}}} \log \frac{\mathrm{P}\left(\theta_{l}\right)}{\mathrm{P}\left(\theta_{l}^{\prime}\right)}+\log \mathrm{P}(\mathbf{Y} \mid \bar{\varphi})-\log \mathrm{P}\left(\mathbf{Y} \mid \bar{\varphi}^{\prime}\right) \\
= & \sum_{l=1}^{N_{\mathrm{T}}} \Lambda_{\theta}\left(\theta_{l}: \theta_{l}^{\prime}\right)-\frac{1}{2 \sigma_{\mathrm{n}}^{2}}|\mathbf{Y}-\mathbf{\Phi}(\bar{\varphi}) \tilde{\mathbf{S}}(\bar{\varphi})|^{2} \\
& +\frac{1}{2 \sigma_{\mathrm{n}}^{2}}\left|\mathbf{Y}-\boldsymbol{\Phi}\left(\bar{\varphi}^{\prime}\right) \tilde{\mathbf{S}}\left(\bar{\varphi}^{\prime}\right)\right|^{2},
\end{aligned}
$$

where a priori information $\Lambda_{\theta}\left(\theta_{l}: \theta_{l}^{\prime}\right)$ is defined as

$$
\begin{aligned}
& \Lambda_{\theta}\left(\theta_{l}: \theta_{l}^{\prime}\right)= \begin{cases}+\Lambda_{\mathrm{a}, l}^{(\theta)} & \left(\theta_{l}=+\frac{\pi}{4}, \theta_{l}^{\prime}=-\frac{\pi}{4}\right) \\
-\Lambda_{\mathrm{a}, l}^{(\theta)} & \left(\theta_{l}=-\frac{\pi}{4}, \theta_{l}^{\prime}=+\frac{\pi}{4}\right), \\
0 & \left(\theta_{l}=\theta_{l}^{\prime}\right)\end{cases} \\
& \Lambda_{\mathrm{a}, l}^{(\theta)}=\log \frac{\mathrm{P}\left(\theta_{l}=+\frac{\pi}{4}\right)}{\mathrm{P}\left(\theta_{l}=-\frac{\pi}{4}\right)},
\end{aligned}
$$

where $\Lambda_{\mathrm{a}, l}^{(\theta)}$ represents a priori information of the $l$ th phase $\theta_{l}^{\dagger}$ that is fed back from the decoder. The a priori information $\Lambda_{\mathrm{a}, l}^{(\theta)}$ is defined in the next section. The optimum phase pattern $\bar{\varphi}_{\text {est }}$ that satisfies the following equation is selected as follows.

$$
\Lambda_{\bar{\varphi}}\left(\bar{\varphi}_{\mathrm{est}}: \bar{\varphi}^{\prime}\right) \geq 0 \text { for } \forall \bar{\varphi}^{\prime} \neq \bar{\varphi}_{\text {est }} .
$$

For the selected phase pattern $\bar{\varphi}_{\text {est }}$, the estimated data signal vector $\mathbf{D}_{\text {det }}\left(\bar{\varphi}_{\text {est }}\right)$ can be calculated according to the definition of $\mathbf{D}$ in (5).

$$
\mathbf{D}_{\text {det }}\left(\bar{\varphi}_{\text {est }}\right)=\boldsymbol{\Omega}\left(\bar{\varphi}_{\text {est }}\right) \mathbf{S}_{\text {det }}\left(\bar{\varphi}_{\text {est }}\right) .
$$

The estimated data vector $\mathbf{D}_{\text {det }}\left(\bar{\varphi}_{\text {est }}\right)$ is output to the Turbo decoder through the deinterleaver. In other words, the proposed receiver provides the Turbo decoder with those output signals via the interleaver. This means that the detector does not output extrinsic information, which is a big difference from Turbo equalizers: a representative of iterative receivers.

Though the definition of the phase is regarded as an application of the principle of the bit LLR, the definition is not equal to that of the bit LLR. This generates the performance shown in Sec.4 


\subsection{3 a priori Information Fed Back to the MIMO Detec-} tor for Iterative Decoding

The Turbo decoder calculates the bit LLR $L_{\mathrm{d}, v}^{(d)}$ in the same way as usual Turbo decoders. The bit LLR is defined as

$$
L_{\mathrm{d}, v}^{(d)}=\log \frac{\mathrm{P}\left(c_{\mathrm{d}, v}=1 \mid \mathbf{Y}\right)}{\mathrm{P}\left(c_{\mathrm{d}, v}=0 \mid \mathbf{Y}\right)},
$$

where $c_{\mathrm{d}, v}$ represents the $v$ th bit. After the $N_{\text {in }}$ iterations of the decoding, the Turbo decoder feeds back the extrinsic bit LLR $L_{\mathrm{ex}, v}$ to the MIMO detector as a priori information $L_{\mathrm{a}, u}$ via the interleaver.

$$
L_{\mathrm{a}, u}=\pi\left[L_{\mathrm{ex}, u}\right],
$$

where $\pi[\bullet]$ represents a function of the interleaver. Whereas the extrinsic bit LLRs are fed back from the Turbo decoder, the MIMO detector requires the a priori information of the real signal and the phase. Therefore, we propose a scheme to convert the extrinsic bit LLRs into the a priori information of the real signals $s_{l}(\bar{\varphi})$ and the phase $\theta_{l}$. The proposed conversion can be obtained by the following derivation.

$$
\begin{aligned}
& \Lambda_{\mathrm{a}, l}^{(s)}(\bar{\varphi})=\log \frac{\mathrm{P}\left(s_{l}(\bar{\varphi})=+\sqrt{2}\right)}{\mathrm{P}\left(s_{l}(\bar{\varphi})=-\sqrt{2}\right)}=\log \frac{\mathrm{P}\left(\mathfrak{R}\left[d_{l}\right]=+1\right)}{\mathrm{P}\left(\mathfrak{R}\left[d_{l}\right]=-1\right)} \\
& =L_{\mathrm{a}, 2 u}, \\
& \Lambda_{\mathrm{a}, l}^{(\theta)}=\log \frac{\mathrm{P}\left(\theta_{l}=+\frac{\pi}{4}\right)}{\mathrm{P}\left(\theta_{l}=-\frac{\pi}{4}\right)} \\
& =\log \frac{\mathrm{P}\left(d_{l}=+1+\mathrm{j}\right)+\mathrm{P}\left(d_{l}=-1-\mathrm{j}\right)}{\mathrm{P}\left(d_{l}=-1+\mathrm{j}\right)+\mathrm{P}\left(d_{l}=+1-\mathrm{j}\right)} \\
& =\log \frac{\frac{\mathrm{P}\left(\mathfrak{R}\left[d_{l}\right]=+1\right) \mathrm{P}\left(\mathfrak{I}\left[d_{l}\right]=+1\right)}{\mathrm{P}\left(\mathfrak{J}\left[d_{l}\right]=-1\right) \mathrm{P}\left(\mathfrak{J}\left[d_{l}\right]=-1\right)}+1}{\mathrm{P}\left(\mathfrak{J}\left[d_{l}\right]=-1\right)}+\frac{\mathrm{P}\left(\mathfrak{R}\left[d_{l}\right]=+1\right)}{\mathrm{P}\left(\mathfrak{R}\left[d_{l}\right]=-1\right)} \\
& =\max \left[L_{\mathrm{a}, 2 u}+L_{\mathrm{a}, 2 u+1}, 0\right]-\max \left[L_{\mathrm{a}, 2 u}, L_{\mathrm{a}, 2 u+1}\right],
\end{aligned}
$$

where $\mathfrak{R}[a]$ and $\mathfrak{I}[a]$ represent a real part and an imaginary part of a complex number $a$, respectively. Note that the $2 u$ th and $(2 u+1)$ th bits correspond to the real part and the imaginary part of the $l$ th QPSK signal, respectively.

\subsection{Procedure of the proposed SHISO receiver}

The MIMO detector estimates the signal vector $\mathbf{D}_{\text {det }}\left(\bar{\varphi}_{\text {est }}\right)$ based on the proposed scheme described in Sect. 3.1. The vector $\mathbf{D}_{\text {det }}\left(\bar{\varphi}_{\text {est }}\right)$ is output to the Turbo decoder, which feeds back the extrinsic bit LLR to the MIMO detector. This operation is iterated $N_{\text {out }}$ times in the proposed receiver. Since the vector $\mathbf{D}_{\text {det }}\left(\bar{\varphi}_{\text {est }}\right)$ is the product of the hard decision rotation matrix $\boldsymbol{\Omega}\left(\bar{\varphi}_{\mathrm{est}}\right)$ and the soft real signal vector $\mathbf{S}_{\mathrm{det}}\left(\bar{\varphi}_{\mathrm{est}}\right)$ as is defined in (31), the vector is called as a semi-hard decision signal vector. This is the reason why the proposed receiver is named as an iterative SHISO receiver. The proposed receiver has the iterative signal processing between the MIMO detector and the Turbo decoder and the iterative processing of the Turbo decoding. To distinguish the two iteration processing. we call the iterative exchange of the signals between the MIMO detector and the Turbo decoder as "outer iteration." $N_{\text {out }}$ denotes the number of the outer iterations. The extrinsic bit LLR is exchanged $N_{\text {in }}$ times between the two component decoder in the Turbo decoder. we call this iteration within the Turbo decoder as "inner iteration". $N_{\text {in }}$ represents the number of the inner iterations.

\subsection{Extended MAP Estimation for Virtual Channel Detec- tion}

The virtual channel is detected based on the MAP in (25), assuming that the real signals in the virtual channel $\boldsymbol{\Phi}(\bar{\varphi})$ are almost the same to those in $\boldsymbol{\Phi}\left(\bar{\varphi}^{\prime}\right)^{\dagger}$. However, the assumption does not always hold true. In this section, we propose a novel estimation criterion for the virtual channel detection where it is taken into account that the real signals in a virtual channel might be different from those in the others. Our proposed estimation criterion applies an LLR $\Lambda_{\mathbf{D}}\left(\bar{\varphi}: \bar{\varphi}^{\prime}\right)$ of the phase pattern $\bar{\varphi}$, which is defined as

$$
\Lambda_{\mathbf{D}}\left(\bar{\varphi}: \bar{\varphi}^{\prime}\right)=\log \frac{\mathrm{P}(\mathbf{Y} \mid \mathbf{D}(\bar{\varphi})) \mathrm{P}(\mathbf{D}(\bar{\varphi}))}{\mathrm{P}\left(\mathbf{Y} \mid \mathbf{D}^{\prime}(\bar{\varphi})\right) \mathrm{P}\left(\mathbf{D}^{\prime}(\bar{\varphi})\right)} .
$$

Because the rotation matrices $\boldsymbol{\Omega}(\bar{\varphi})$ and the real signal vectors $\mathbf{S}(\bar{\varphi})$ can be assumed to be independent from each other, the LLR of the phase pattern, $\Lambda_{\mathbf{D}}\left(\bar{\varphi}: \bar{\varphi}^{\prime}\right)$, can be rewritten using (5) as

$$
\begin{aligned}
\Lambda_{\mathbf{D}}\left(\bar{\varphi}: \bar{\varphi}^{\prime}\right)= & \log \frac{\mathrm{P}(\mathbf{Y} \mid \boldsymbol{\Omega}(\bar{\varphi}) \tilde{\mathbf{S}}(\bar{\varphi})) \mathrm{P}(\boldsymbol{\Omega}(\bar{\varphi})) \mathrm{P}(\tilde{\mathbf{S}}(\bar{\varphi}))}{\mathrm{P}\left(\mathbf{Y} \mid \mathbf{\Omega}\left(\bar{\varphi}^{\prime}\right) \mathbf{S}^{\prime}(\bar{\varphi})\right) \mathrm{P}\left(\boldsymbol{\Omega}\left(\bar{\varphi}^{\prime}\right)\right) \mathrm{P}\left(\mathbf{S}^{\prime}(\bar{\varphi})\right)} \\
= & \sum_{l}^{N_{\mathrm{T}}} \log \frac{\mathrm{P}\left(\theta_{l}\right)}{\mathrm{P}\left(\theta_{l}^{\prime}\right)}+\sum_{l}^{N_{\mathrm{T}}} \log \frac{\mathrm{P}\left(s_{l}(\bar{\varphi})\right)}{\mathrm{P}\left(s_{l}^{\prime}(\bar{\varphi})\right)} \\
& +\log \mathrm{P}(\mathbf{Y} \mid \mathbf{\Omega}(\bar{\varphi}) \tilde{\mathbf{S}}(\bar{\varphi})) \\
& -\log \mathrm{P}\left(\mathbf{Y} \mid \mathbf{\Omega}\left(\bar{\varphi}^{\prime}\right) \mathbf{S}^{\prime}(\bar{\varphi})\right) \\
= & \sum_{l}^{N_{\mathrm{T}}} \Lambda_{\theta}\left(\theta_{l}: \theta_{l}^{\prime}\right)+\sum_{l}^{N_{\mathrm{T}}} \Lambda_{s}\left(s_{l}: s_{l}^{\prime}\right) \\
& -\frac{1}{2 \sigma_{\mathrm{n}}^{2}}|\mathbf{Y}-\boldsymbol{\Phi}(\bar{\varphi}) \tilde{\mathbf{S}}(\bar{\varphi})|^{2} \\
& +\frac{1}{2 \sigma_{\mathrm{n}}^{2}}\left|\mathbf{Y}-\mathbf{\Phi}\left(\bar{\varphi}^{\prime}\right) \mathbf{S}^{\prime}(\bar{\varphi})\right|^{2}, \\
\Lambda_{s}\left(s_{l}: s_{l}^{\prime}\right)= & \begin{cases}+\Lambda_{\mathrm{a}, l}^{(s)} & \left(s_{l}=+\sqrt{2}, s_{l}^{\prime}=-\sqrt{2}\right) \\
-\Lambda_{\mathrm{a}, l}^{(s)} & \left(s_{l}=-\sqrt{2}, s_{l}^{\prime}=+\sqrt{2}\right) \\
0 & \left(s_{l}=s_{l}^{\prime}\right)\end{cases}
\end{aligned}
$$

†This assumption is also applied in [7], [9]. The proposed receiver explained in Sec.3.1 attains only slightly better performance than the receiver proposed in [9], which is described in Sec.4.2, even though the Turbo code is applied in the proposed receiver. We think that the application of this assumption limits the performance gain. 
where $\Lambda_{\mathrm{a}, l}^{(s)}$ represents a priori information of the real signal defined in (34). As is similar to (30), the optimum phase pattern $\bar{\varphi}_{\text {est }}$ that satisfies the following equation is selected:

$$
\Lambda_{\mathbf{D}}\left(\bar{\varphi}_{\mathrm{est}}: \bar{\varphi}^{\prime}\right) \geq 0 \text { for } \forall \bar{\varphi}^{\prime} \neq \bar{\varphi}_{\mathrm{est}} .
$$

Then, the optimum signal vector $\mathbf{D}_{\text {det }}\left(\bar{\varphi}_{\text {est }}\right)$ is calculated as

$$
\mathbf{D}_{\text {det }}\left(\bar{\varphi}_{\text {est }}\right)=\boldsymbol{\Omega}\left(\bar{\varphi}_{\text {est }}\right) \mathbf{S}_{\text {det }}\left(\bar{\varphi}_{\text {est }}\right) .
$$

This optimum signal vector is output to the Turbo decoder through the deinterleaver. Because not only $\Lambda_{\theta}\left(\theta_{l}: \theta_{l}^{\prime}\right)$ but also $\Lambda_{s}\left(s_{l}: s_{l}^{\prime}\right)$ are taken into account in the virtual channel detection, the proposed detection is expected to achieve better performance than the technique explained in Sect. 3.1.2.

\section{Simulation Results}

In this section, we evaluate the BER performance of the proposed receiver by computer simulation. Simulation parameters are listed in Table 1. The channel impulse responses are independently and identically distributed (i.i.d.) and are quasi-static. In addition, the detector is assumed to have the perfect channel state information (CSI). We apply the Turbo code introduced in [10]. The Max-log MAP algorithm is applied to all the MAP estimations in this paper.

\subsection{SHISO Iterative Receiver}

Figure 3 shows the BER performance of the proposed receiver with some combinations of the inner iterations and the outer iterations, i.e., $N_{\text {in }}$ and $N_{\text {out }}$. However, $N_{\text {out }} \times N_{\text {in }}$ is kept to 48 in all the combinations. In addition, the performance of the SISO iterative receiver composed of the

Table 1 Simulation parameters.

\begin{tabular}{c|c}
\hline$N_{\mathrm{T}} \times N_{\mathrm{R}}$ & $6 \times 2$ \\
Modulation scheme & OFDM-QPSK \\
Channel model & 10-path Rayleigh fading \\
Forward error correction & Turbo code, rate $1 / 3, K=4$ \\
No. of sub carriers & 512 \\
Data packet length & 16384 bits \\
\hline
\end{tabular}

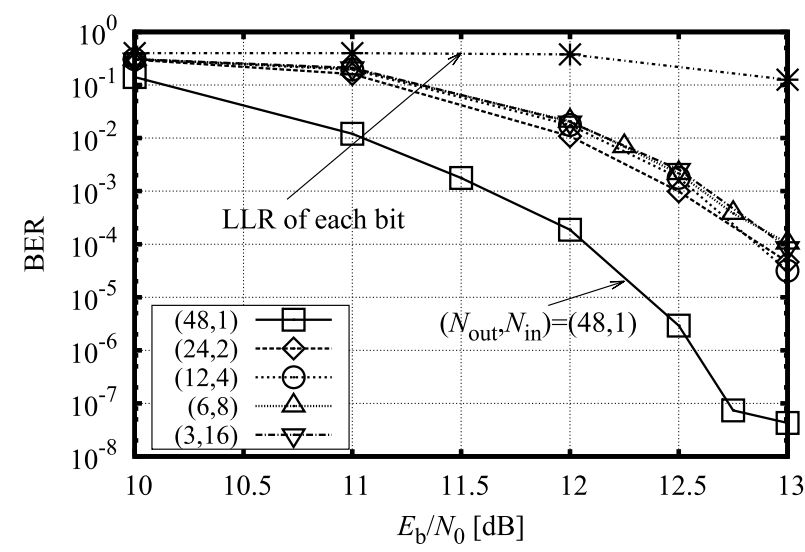

Fig. 3 BER performance of the proposed receiver.
MIMO detector and the Turbo decoder is added, which is labelled as "LLR of each bit". In the SISO iterative receiver, a technique written in Appendix to implement a soft input soft output iteration receiver is applied ${ }^{\dagger}$. The setting of $\left(N_{\text {out }}, N_{\text {in }}\right)$ is $(6,8)$ in the SISO iterative receiver. Obviously, the performance of the SISO iterative receiver is much worse than that of the proposed receiver. The reason is discussed below. It is shown that the BER performance with $\left(N_{\text {out }}, N_{\text {in }}\right)=(48,1)$ is better than the performance with $(24,2)$ by about $0.9 \mathrm{~dB}$ at BER $=10^{-5}$. The reason is also discussed in the following.

Figure 4 shows the cumulative distribution function (CDF) of misestimated extrinsic bit LLR that is fed back from the Turbo decoder to the MIMO detector. The misestimated extrinsic bit LLR is defined as the extrinsic bit LLR whose sign is different from that of the transmitted bit. Since the extrinsic bit LLR multiplied by the corresponding transmitted bit is shown in the figure, the sign of the misestimated extrinsic bit LLR is only minus. In the figure, the plot with " $\left(N_{\text {out }}, N_{\text {in }}\right)$, end" shows the CDF of the misestimated extrinsic bit LLR at the end of all the iterations, while the plot with " $\left(N_{\text {out }}, N_{\text {in }}\right)$, begin" shows the CDFs of misestimated extrinsic bit LLR at the beginning of the iterations.

Because an extrinsic bit LLR is dealt as reliability in the MAP estimation, in principle, a misestimated extrinsic bit LLR that is not reliable, should be close by zero, e.g., $L_{\mathrm{ex}, v}=\log \frac{\mathrm{P}\left(\mathfrak{R}\left[d_{l}\right]=+1\right)}{\mathrm{P}\left(\mathfrak{R}\left[d_{l}\right]=-1\right)} \approx 0$ in the proposed receiver. If a misestimated extrinsic bit LLR with a big absolute value is provided to the MIMO detector, performance of the proposed detector is not improved enough. As is shown in Fig. 4, the distribution of the misestimated bit LLR has a bigger absolute value with relatively higher probability. Because the SISO iterative receiver circulates the misestimated extrinsic bit LLRs between the MIMO detector and the Turbo decoder. the misestimated extrinsic bit LLR with a big absolute value not only deteriorates the signal detection at

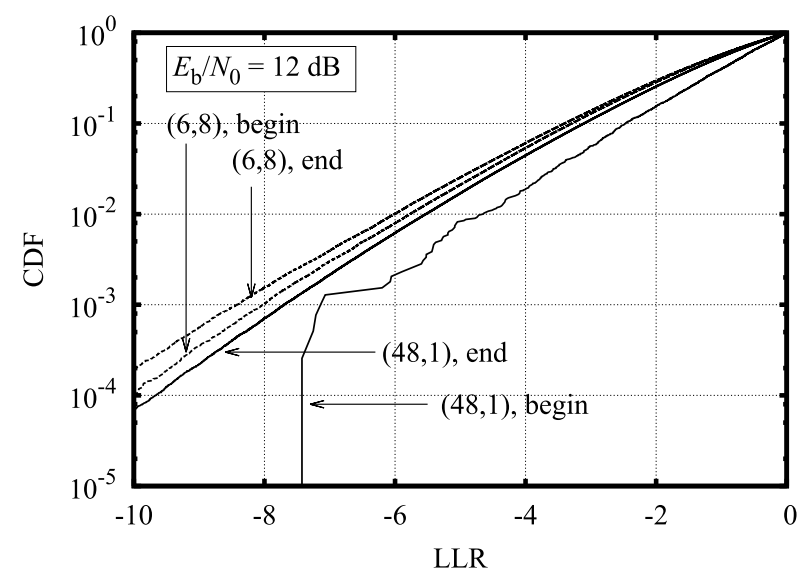

Fig. 4 CDF of misestimated extrinsic bit LLR.

The technique to calculate the LLR is not a standard technique, but is derived for the iterative MIMO receiver consisting of the MIMO detector with virtual channels and the decorders. 
the MIMO detector, but also degrades the Turbo decoding. Though the performance shown in Fig. 4 is not that of the SISO receiver, the similar performance probably appears at the decoder output signals, because only the extrinsic LLRs only replace the semi-hard decision signals in the SISO receiver. The performance of the SISO receiver can be explained with the performance in Fig. 4. Anyway, the misestimated extrinsic bit LLRs degrades the SISO iterative receiver for the reason explained above.

As is described above, the MIMO detector outputs the hard decision signals associated with the phase to the Turbo decoder in the proposed SHISO receiver. When the misestimated extrinsic bit LLRs are fed back to the MIMO detector, the misestimated bits are included in the hard decision signals with a certain probability due to the wrong feedback information. However, since the amplitude of the hard decision signals is rounded to 1 that is is much smaller than that from misestimated extrinsic bit LLR, the damage from the misestimated bits is limited in the proposed SHISO receiver, especially, in the Turbo decoder. As is shown in Fig. 4, for instance, the absolute value of the misestimated bit LLR happens to reach to 10 , which is much bigger than 1 . Therefore, the proposed SHISO iterative receiver achieves better performance than the SISO iterative receiver, which is proven by Fig. 3 where the SISO iterative receiver is labelled as "LLR of each bit". Generally speaking, SISO iterative receivers are well-known to achieve the best performance when the distribution of the input signal is Gaussian. However, when the misestimated extrinsic bit LLR has distribution that the big value appears at high probability, SISO iterative receivers is not always optimum and might has worse performance than HISO iterative receivers.

On the other hand, the misestimated extrinsic bit LLR with $\left(N_{\text {out }}, N_{\text {in }}\right)=(48,1)$ is smaller than that with the others at the end of the iteration. This means that no inner iteration, i.e., $N_{\text {in }}=1$, reduces the possibility to feed back the misestimated extrinsic bit LLR with a big absolute value to the decoder. This is the reason why the proposed receiver with $N_{\text {in }}=1$ achieves better performance than that with $N_{\text {in }}>1$ as shown in Fig. 3 .

\subsection{Receiver with Proposed Extended MAP Estimation}

Figure 5 shows the BER performance when the extended MAP estimation explained in Sec.3.3 is applied. The BER performance with $\left(N_{\text {out }}, N_{\text {in }}\right)=(48,1)$ is better than that with $(24,2)$ by about $2.5 \mathrm{~dB}$ at BER $=10^{-5}$. Apparently, the proposed receiver with $N_{\text {in }}=1$ achieves the best performance in the other combinations, while Turbo decoders usually improve decoding performance by increasing the number of turbo iterations that corresponds to that of the inner iterations. Whereas the inner iteration does not seem to contribute to the performance improvement, the outer iteration makes the receiver achieve such a performance. The outer iteration plays an important role to attain such a performance, even though some signal processing is included in the outer iteration. The reason is also discussed below.

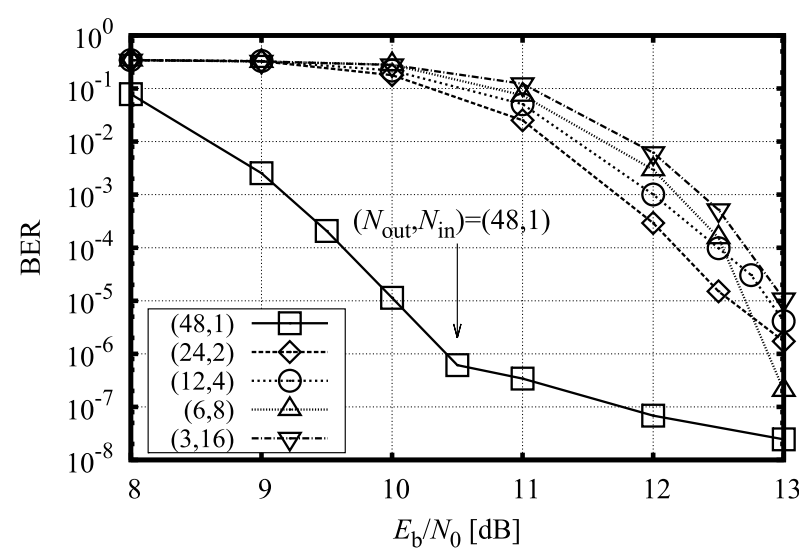

Fig. 5 BER performance with the extended MAP estimation.

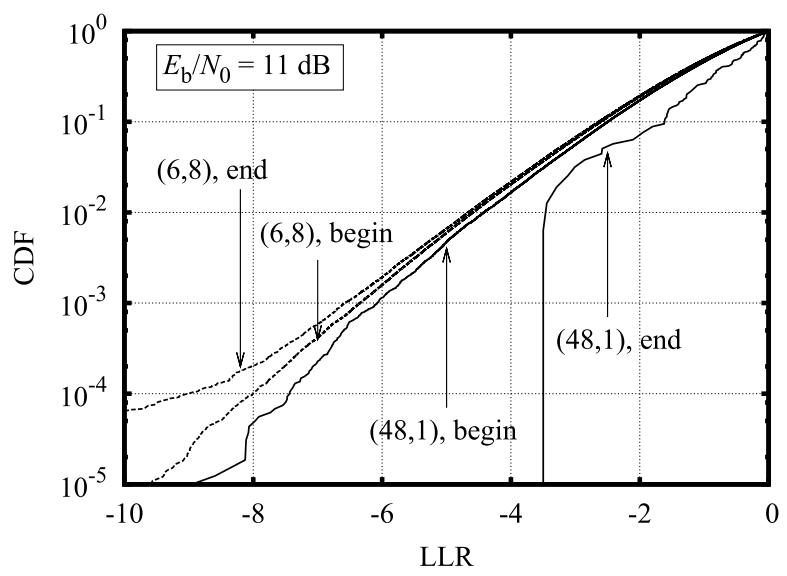

Fig. 6 CDF of misestimated extrinsic bit LLR.

Figure 6 shows the CDF of the misestimated extrinsic bit LLR that is fed back from the Turbo decoder to the MIMO detector. Similar to Fig. 4, the misestimated extrinsic bit LLR with $\left(N_{\text {out }}, N_{\text {in }}\right)=(48,1)$ is much smaller than the others at the end of the iterations. Hence, the performance shown in Fig. 6 strongly supports that the proposed receiver with $\left(N_{\text {out }}, N_{\text {in }}\right)=(48,1)$ achieves better performance than that with $N_{\text {in }} \geq 2$. While the misestimated bit LLRs at the end of the iterations are bigger than those at the begging in the Fig. 4 , however, only the LLR with $\left(N_{\text {out }}, N_{\text {in }}\right)=(48,1)$ at the end of the iterations is smaller than that at the begging in Fig. 6. The reason could be considered as follows.

Because the estimation criterion with the LLR in (25) can not calculate the marginal distribution with high precision, the iterative SHISO MIMO detector with the LLR outputs the data vector $\mathbf{D}_{\text {det }}\left(\bar{\varphi}_{\text {est }}\right)$ that has error symbols associated with the misestimated extrinsic bit LLRs. When the data vector is provided to the Turbo decoder, the probability that the Turbo decoder enhances the absolute values of the misestimated extrinsic bit LLRs is increased. On the other hand, because the extended MAP estimation with the LLR in (36) estimates the marginal distribution with higher accuracy, the MIMO detector based on the extended MAP estimation generates the data vector $\mathbf{D}_{\text {det }}\left(\bar{\varphi}_{\text {est }}\right)$ in which the 


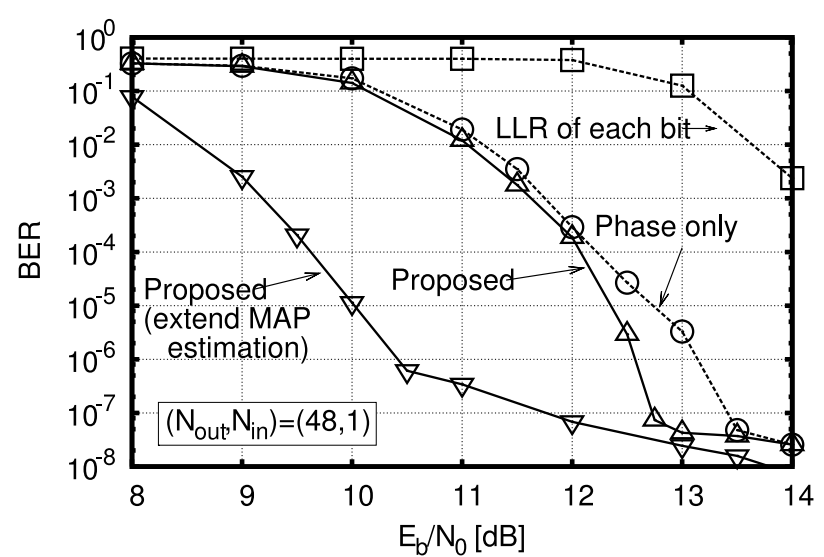

Fig. 7 Performance comparison with Turbo coded detectors.

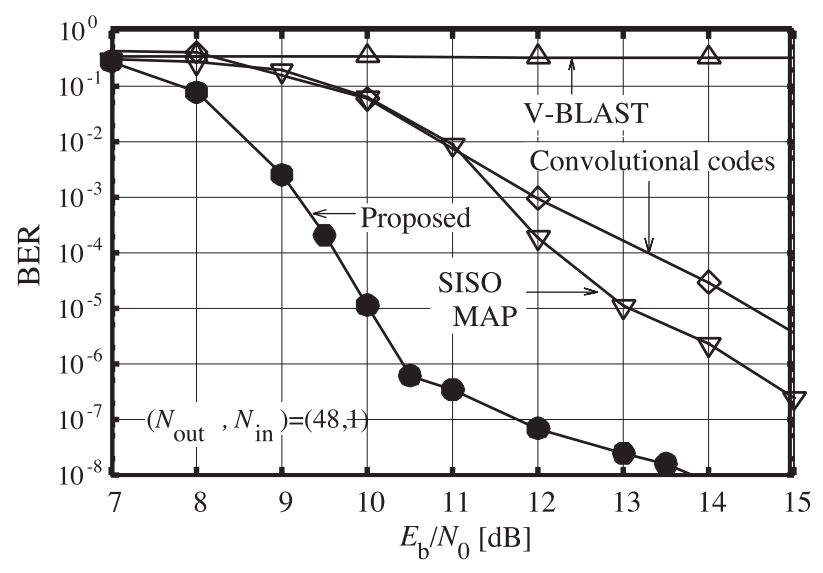

Fig. 8 Performance comparison with conventional detectors.

formance than the receiver with only the phase information by approximately $0.5 \mathrm{~dB}$ at $\mathrm{BER}=10^{-6}$. Moreover, the proposed receiver with the extended MAP estimation achieves a gain of $2.5 \mathrm{~dB}$ at $\mathrm{BER}=10^{-5}$, compared with the proposed receiver.

The BER performance of the proposed receiver with the extended MAP estimation is compared with that of the SISO MAP iterative receiver with the Turbo code, the vertical Bell laboratories layered space-time (V-BLAST) [18] with the same code, and the iterative SISO receiver with the convolutional code [9] in Fig. 8. In the figure, the SISO MAP iterative receiver with the Turbo code and the iterative SISO receiver with convolutional code are labelled as "SISO MAP" and "Convolutional code", respecitively. In the SISO MAP iterative receiver, the MAP detection with brute force search is performed. For fair comparison, the extrinsic bit LLR is circulated between the MAP detector and the Turbo decoder in the SISO MAP iterative receiver.

As is well-known, when the number of the receive antennas is less than that of the streams, linear MIMO detectors such as the V-BLAST, do not have capability to detect the signal streams due to lack of the number of degrees of freedom. Even though the Turbo decoder follows the VBLAST detector, the performance is much worse than the others. The performance of the V-BLAST has an irreducible error at BER of 0.5 .

The iterative SISO receiver with the convolutional code [9] achieves much better performance than the V-BLAST, because the non-linear signal detection is performed in addition to the linear detection in the the receiver. However, the performance of the iterative receiver is inferior to the SISO MAP iterative receiver.

The proposed receiver with the extended MAP estimation achieves furthermore better performance than the SISO MAP iterative receiver by $2.7 \mathrm{~dB}$ at $\mathrm{BER}=10^{-5}$, surprisingly. In principle, the MAP achieves the best performance when the misestimated bit LLR comes close by zero owing to low reliability of the bit. However, some misestimated bit LLRs grow high when the Turbo decoder is applied as is shown above. This degrades the SISO MAP iterative re- ceivers drawn in Fig. 7 are selected as reference receivers, because they have been regarded as reference receivers in MIMO systems. 


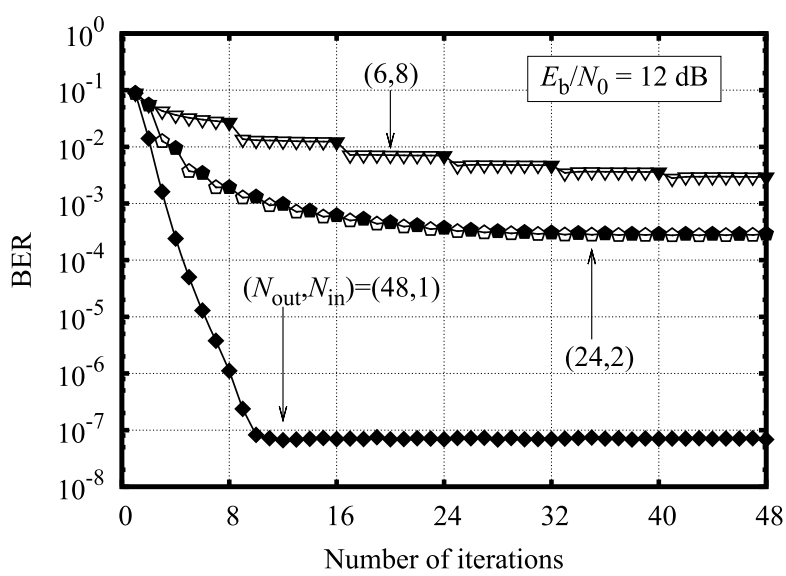

Fig. 9 BER convergence performance of the proposed receiver.

ceiver. Anyway, the proposed receiver with the extended MAP estimation attains a gain of $3.0 \mathrm{~dB}$ at $\mathrm{BER}=10^{-5}$, compared with the iterative receiver with the convolutional code [9]. Performance comparison with various other receivers, e.g., the SISO MAP receiver with the same Turbo decoder in 64QAM $2 \times 2$ MIMO is one of our future tasks.

As is shown in Fig. 8. the BER performance of the proposed receiver is steeper than that of the conventional receiver. The steep BER performance is typical to iterative decoding such as Turbo decoding, even though $\left(N_{\text {out }}, N_{\text {in }}\right)=$ $(48,1)$; the turbo iteration as the inner iteration is not performed. In principle, such a performance can not be obtained without a Turbo decoder, which corresponds to the inner decoder in the propose receiver. Although the inner iteration is not useful, hence, the Turbo decoder as the inner decoder plays a crucial role to improve the performance.

\subsection{Convergence Performance}

The BER convergence performances are shown in Fig. 9 at $E_{\mathrm{b}} / N_{0}=12 \mathrm{~dB}$. The white points $(\circ, \triangle, \square)$ indicate the BER performance of the Turbo decoder outputs when the inner iteration is once finished. The black points $(\bullet, \boldsymbol{\Lambda}, \boldsymbol{\square})$ indicate the BER performance when the outer iteration is once finished. However, some circles are omitted for clear view. It is obviously shown that the BER convergence with $N_{\text {in }}=1$ is faster than that with $N_{\text {in }}>1$.

\subsection{Computational Complexity}

The number of multiplications and additions performed in the MIMO detectors are compared with those in the SISO MAP iterative receiver in Fig. 10, and Fig. 11, respectively. When $\left(N_{\text {out }}, N_{\text {in }}\right)=(48,1)$, the number of multiplications in the proposed receiver is $30.3 \%$ of that required in the SISO MAP iterative receiver and the number of additions required in the proposed receiver is $3.2 \%$ of that in the other. Therefore, it can be said that the proposed receiver is less complex than the SISO MAP iterative receiver.

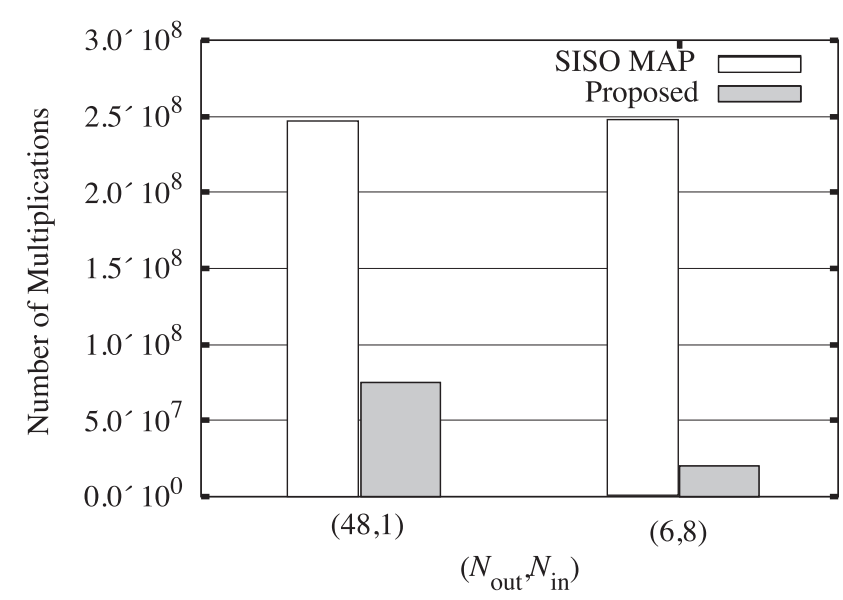

Fig. 10 Complexity in terms of the number of multiplications.

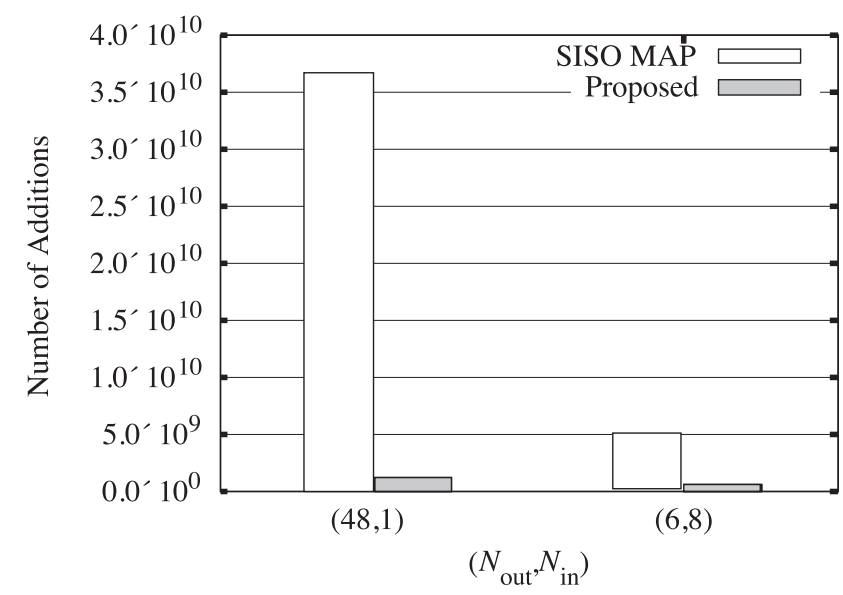

Fig. 11 Complexity in terms of the number of additions.

\section{Conclusions}

This paper proposes a novel receiver called as a "iterative SHISO receiver employing virtual channels with a Turbo decoder" for MIMO-OFDM systems where the number of spatially multiplexed streams is more than twice as many as that of the receive antennas, e.g., $\left(N_{\mathrm{T}}, N_{\mathrm{R}}\right)=(6,2)$. In the proposed iterative SHISO receiver, the extrinsic bit LLR is fed back from the decoder to the MIMO detector as an $a$ priori information, and the real signal and the hard decision signal of the phase are provided to the Turbo decoder. Moreover, this paper proposes a new estimation criterion, named as "extended MAP estimation", for the MIMO detector to select the most likely virtual channel.

The performance of the proposed receiver is verified in a $6 \times 2$ MIMO system by computer simulation. If the product of the number of the outer iterations and that of the inner iterations is set to 48 , the proposed receiver with the inner iteration $N_{\text {in }}$ equal to 1 achieves better performance than that with $N_{\text {in }}>1$. Even when the extended MAP estimation is applied to the proposed receiver, the combination of $\left(N_{\text {out }}, N_{\text {in }}=(48,1)\right.$ makes the receiver achieve the 
best performance in that with the other combinations. In other words, the inner iteration does not seem to contribute to the performance improvement and the outer iteration enables the receiver to achieve the steep BER performance. The outer iteration plays a role of Turbo iteration of Turbo decoding, even though some signal processing is included in the outer iteration. Because such a steep BER performance is typical to iterative decoding such as Turbo decoding, in principle, such a performance can not be obtained without a Turbo decoder, which corresponds to the inner decoder in the propose receiver. Although the inner iteration is not useful, hence, the Turbo decoder as an inner decoder plays a crucial role to improve the performance.

The proposed receiver with the extended MAP estimation achieves better performance than the original SHISO MIMO receiver by $2.5 \mathrm{~dB}$ at BER $=10^{-5}$. The proposed receiver with the extended MAP estimation attains by $3.0 \mathrm{~dB}$ better performance than the SISO MAP iterative receiver at $\mathrm{BER}=10^{-5}$, because the misestimated extrinsic bit LLR provided by the detector deteriorates the Turbo decoding in the SISO MAP iterative receiver. Besides, the proposed receiver has lower computational complexity than the SISO MAP iterative receiver. In summary, the proposed receiver achieves better transmission performance with relatively low computational complexity compared with the SISO MAP iterative receiver. Performance comparison with various other receivers, e.g., the SISO MAP receiver with the same Turbo decoder in 64QAM $2 \times 2$ MIMO is one of our future tasks.

\section{Acknowledgments}

This research is supported by Strategic Information and Communication R\&D Promotion Program (SCOPE) conducted by the Ministry of Internal Affairs and Communication (MIC) of Japan.

\section{References}

[1] R. Chang and R. Gibby, "A theoretical study of performance of an orthogonal multiplexing data transmission scheme," IEEE Trans. Commun., vol.16, no.4, pp.529-540, Aug. 1968.

[2] G.J. Foschini and M.J. Gans, "On limits of wireless communications in a fading environment when using multiple antennas," Wireless Pers. Commun., vol.6, no.3, pp.311-335, March 1998.

[3] A. Goldsmith, S.A. Jafar, N. Jindal, and S. Vishwanath, "Capacity limits of MIMO channels," IEEE J. Sel. Areas Commun., vol.21, no.5, pp.684-702, June 2003.

[4] H. Kawai, K. Higuchi, N. Maeda, and M. Sawahashi, "Adaptive control of surviving symbol replica candidates in QRM-MLD for OFDM MIMO multiplexing," IEEE J. Sel. Areas Commun., vol.24, no.6, pp.1130-1140, June 2006.

[5] K.-K. Wong, A. Paulraj, and R.D. Murch, "Efficient highperformance decoding for overloaded MIMO antenna systems," IEEE Trans. Wireless Commun., vol.6, no.5, pp.1833-1843, May 2007.

[6] B.W. Zarikoff, J.K. Cavers, and S. Bavarian "An iterative groupwise multiuser detector for overloaded MIMO applications," IEEE Trans. Wireless Commun., vol.6, no.2, pp.443-447, Feb. 2007.

[7] S. Denno, H. Maruyama, D. Umehara, and M. Morikura, "A virtual layered space time receiver with maximum likelihood channel detection," Proc. IEEE VTC'09 Spring, pp.1-5, Barcelona, April 2009.

[8] S. Yoshikawa, S. Denno, and M. Morikura, "Complexity reduced lattice-reduction-aided MIMO receiver with virtual channel detection,” IEICE Trans. Commun., vol.E96-B, no.1, pp.263-270, Jan. 2013.

[9] J. Imamura, S. Denno, D. Umehara, and M. Morikura, "A virtual layered space-frequency receiver architecture with iterative decoding," IEICE Trans. Commun., vol.E94-B, no.7, pp.1994-2002, July 2011.

[10] C. Berrou and A. Glavieux, "Near optimum error correcting coding and decoding: Turbo-codes," IEEE Trans. Commun., vol.44, no.10, pp.1261-1271, Oct. 1996.

[11] H. Kawai, K. Higuchi, N. Maeda, and M. Sawahashi, "Likelihood function for QRM-MLD suitable for soft-decision Turbo decoding and its performance for OFCDM MIMO multiplexing in multipath fading channels," IEICE Trans. Commun., vol.E88-B, no.1, pp.4757, Jan. 2005.

[12] A. Stefanov and T.M. Duman, "Turbo-coded modulation for systems with transmit and antenna diversity over block fading channels: System model, decoding approaches, and practical considerations," IEEE J. Sel. Areas Commun., vol.19, no.5, pp.958-968, May 2001.

[13] B.M. Hochwald and S. t. Brink, "Achieving near-capacity on a multiple-antenna channel," IEEE Trans. Commun., vol.51, no.3, pp.389-399, March 2003.

[14] J. Ylioinas and M. Juntti, "Iterative joint detection, decoding, and channel estimation in turbo-coded MIMO-OFDM," IEEE Trans. Veh. Technol., vol.58, no.4, pp.1784-1796, May 2009.

[15] C. Douillard, C.B.M. Jezequel, C. Berrou, A. Picart, P. Didier and A. Glavieux, "IIterative correction of intersymbol interference: Turboequalisation," European Trans. Telecommun., vol.6, no.5, pp.507-511, Sept. 1995.

[16] X. Wang and H.V. Poor, "IIterative (turbo) soft interference cancellation and decoding for coded CDMA," IEEE Trans. Commun., vol.47, no.7, pp.1046-1061, July 1999.

[17] H.E. Gamal and E. Geraniotis, "IIterative multiuser detection for coded CDMA signals in AWGN and fading channels," IEEE J. Sel. Areas Commun., vol.18, no.1, pp.30-41, Jan. 2000.

[18] G.J. Foschini, "Layered space-time architecture for wireless communication in a fading environment when using multi-element antennas," Bell Labs Tech. J., vol.1, no.2, pp.41-59, 1996.

\section{Appendix: LLRs of Real Signals and Phases}

The iterative detector with virtual channels calculates the LLRs of both the real signal and the phase in order to output soft signals to the Turbo decoder. First, the detector obtain the LLR of the phase $\lambda_{l}^{(\theta)}$, using the Max-Log-MAP approximation, $\lambda_{l}^{(\theta)}$.

$$
\begin{aligned}
\Lambda_{l}^{(\theta)}= & \log \frac{P\left(\theta_{l}=+\frac{\pi}{4} \mid \mathbf{Y}\right)}{P\left(\theta_{l}=-\frac{\pi}{4} \mid \mathbf{Y}\right)} \\
= & \Lambda_{\mathrm{a}, l}^{(\theta)}+\max _{\theta_{l}=+\frac{\pi}{4}}\left[\sum_{m \neq l}^{N_{\mathrm{T}}} \Lambda_{\mathrm{a}, m}^{(\theta)}+J(\bar{\varphi})\right] \\
& -\max _{\theta_{l}=-\frac{\pi}{4}}\left[\sum_{m \neq l}^{N_{\mathrm{T}}} \Lambda_{\mathrm{a}, m}^{(\theta)}+J(\bar{\varphi})\right], \\
J(\bar{\varphi})= & -\frac{1}{2 \sigma_{\mathrm{n}}^{2}}|\mathbf{Y}-\boldsymbol{\Phi}(\bar{\varphi}) \tilde{\mathbf{S}}(\bar{\varphi})|^{2},
\end{aligned}
$$

where $\Lambda_{\mathrm{a}, l}^{(\theta)}$ represents the a priori information of the phase $\theta_{l}$ 
fed back from the Turbo decoder via the converter defined in (35) The LLR of the phase is provided to an slicer to extract only the sign from the LLR. When the sign is positive, $\theta_{l}=$ $\pi / 4$ is estimated as a transmitted phase. Otherwise, $\theta_{l}=$ $-\pi / 4$ is estimated. This processing is regarded as a hard decision. After the processing is applied for all the phases $\theta_{l} l=1, . ., N_{\mathrm{T}}$, the most likelihood virtual channel can be estimated by using (5), (6), and (10).

Next, the LLR of the real signal in the most likelihood virtual channel is calculated as

$$
\begin{aligned}
& \Lambda_{l}^{(s)}\left(\bar{\varphi}_{\mathrm{est}}\right)=\log \frac{P\left(s_{l}=+\sqrt{2} \mid \mathbf{Y}\right)}{P\left(s_{l}=-\sqrt{2} \mid \mathbf{Y}\right)} \\
& =\Lambda_{\mathrm{a}, l}^{(s)}+\max _{\phi \in s_{l}=+\sqrt{2}}\left[\sum_{m \neq l}^{N_{\mathrm{T}}} \Lambda_{\mathrm{a}, m}^{(s)}+J_{s}(\phi)\right] \\
& -\max _{\phi \in s_{l}=-\sqrt{2}}\left[\sum_{m \neq l}^{N_{\mathrm{T}}} \Lambda_{\mathrm{a}, m}^{(s)}+J_{s}(\phi)\right], \\
& J_{s}(\phi)=-\frac{1}{2 \sigma_{\mathrm{n}}^{2}}\left|\mathbf{Y}-\boldsymbol{\Phi}\left(\bar{\varphi}_{\text {est }}\right) \mathbf{S}(\phi)\right|^{2} \text {. }
\end{aligned}
$$

In (A.4), $\bar{\varphi}_{\text {est }}$ is the phase pattern associated with the most likelihood virtual channel. In addition, $\phi$ represents the set of the real signals; $\mathbf{S}(\phi) \phi \in s_{l}=+\sqrt{2}$ means the set of the real signal vectors having $s_{l}=+\sqrt{2}$ as the $l$ th entry.

The MIMO detector outputs the extrinsic bit LLR to the Turbo decoder. The extrinsic bit LLR is defined as

$$
\begin{aligned}
& \Lambda_{\mathrm{ex}, l}^{(s)}=\Lambda_{l}^{(s)}\left(\bar{\varphi}_{\mathrm{est}}\right)-\Lambda_{\mathrm{a}, l}^{(s)}, \\
& \Lambda_{\mathrm{ex}, l}^{(\theta)}=\Lambda_{l}^{(\theta)}-\Lambda_{\mathrm{a}, l}^{(\theta)} .
\end{aligned}
$$

This LLR is converted to the bit LLR and then inputted into the Turbo decoder.

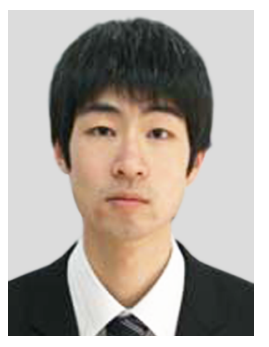

Akihito Taya received his B.E. degree from Kyoto University, Japan, in 2011. He graduated from graduate school of informatics, Kyoto University in 2013. He is now with Hitachi, Ltd. He has a considerable interest in mobile radio communications, especially in spatial multiplexing. $\mathrm{He}$ is a member of the IEICE and a member of the IEEE.

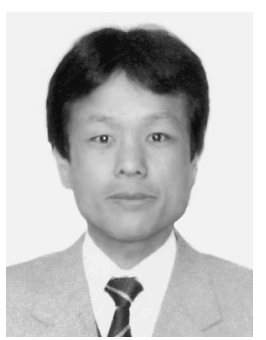

Satoshi Denno received the M.E. and Ph.D. degrees from Kyoto University, Kyoto, Japan in 1988 and 2000, respectively. He joined NTT radio communications systems labs, Yokosuka, Japan, in 1988. In 1997, he was seconded to ATR adaptive communications research laboratories, Kyoto, Japan. From 2000 to 2002, he worked for NTT DoCoMo, Yokosuka, Japan. In 2002, he moved to DoCoMo communications laboratories Europe $\mathrm{GmbH}$, Germany. From 2004 to 2011, he worked as an associate professor at Kyoto University. Since 2011, he is a full professor at graduate school of natural science and technology, Okayama University. From the beginning of his research career, he has been engaged in the research and development of digital mobile radio communications. In particular, he has considerable interests in channel equalization, array signal processing, Space time codes, spatial multiplexing, and multimode reception. He received the Excellent Paper Award from the IEICE in 1995.

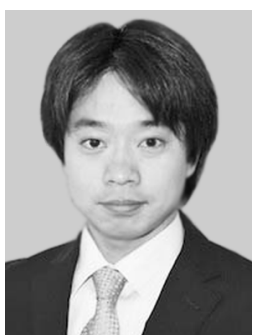

Koji Yamamoto received the B.E. degree in electrical and electronic engineering from Kyoto University in 2002, and the M.E. and Ph.D. degrees in informatics from Kyoto University in 2004 and 2005, respectively. From 2004 to 2005, he was a Research Fellow of the Japan Society for the Promotion of Science (JSPS). Since 2005, he has been with the Graduate School of Informatics, Kyoto University, where he is currently an Associate Professor. From 2008 to 2009, he was a Visiting Researcher at Wireless@KTH, Royal Institute of Technology (KTH) in Sweden. His research interests include game theory, spectrum sharing, and cooperative multi-hop networks. He received the PIMRC 2004 Best Student Paper Award in 2004, the Ericsson Young Scientist Award in 2006, and the Young Researcher's Award from the IEICE of Japan in 2008. He is a member of the IEEE

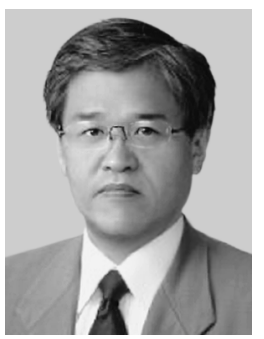

Masahiro Morikura received the B.E., M.E., and Ph.D. degrees in electronics engineering from Kyoto University, Kyoto, Japan in 1979, 1981, and 1991, respectively. $\mathrm{He}$ joined NTT in 1981, where he was engaged in the research and development of TDMA equipment for satellite communications. From 1988 to 1989 , he was with the communications Research Centre, Canada, as a guest scientist. From 1997 to 2002, he was active in the standardization of the IEEE802.11a based wireles LAN. He received the Paper Award and the Achievement Award from IEICE in 2000 and 2006, respectively. He also received the Education, Culture, Sports, Science and Technology Minister Award in 2007. Dr. Morikura is now a Full Professor in the Graduate School of Informatics, Kyoto University. He is a member of IEEE. 


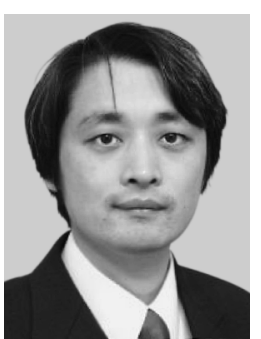

IEEE.

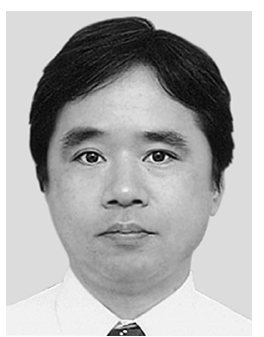

Hidekazu Murata received the B.E., M.E., and Ph.D. degrees in electronic engineering from Kyoto University, Kyoto, Japan, in 1991, 1993, and 2000, respectively. In 1993, he joined the Faculty of Engineering, Kyoto University. From 2002 to 2006, he was an Associate Professor at the Tokyo Institute of Technology. He has been at Kyoto University since October 2006 and is currently an Associate Professor in the Department of Communications and Computer Engineering, Graduate School of Informatics. His major research interests include signal processing and its hardware implementation, with particular application to cooperative wireless networks. He received the Young Researcher's Award from the IEICE of Japan in 1997, the Ericsson Young Scientist Award in 2000, the Young Scientists' Prize of the Commendation for Science and Technology by the Minister of Education, Culture, Sports, Science and Technology in 2006, and the Paper Award of the IEICE in 2011 and 2013, and IEE ICC best paper award in 2014. He is a member of the IEEE.

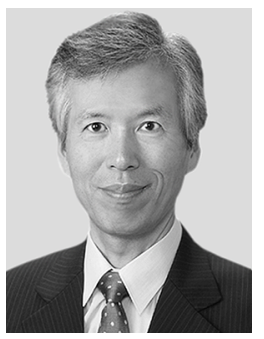

Susumu Yoshida received the B.E., M.E., and $\mathrm{Ph} . \mathrm{D}$. degrees, all in electrical engineering, from Kyoto University, Kyoto, Japan, in 1971, 1973, and 1978, respectively. From 1973, he had been with the Faculty of Engineering, Kyoto University and was a full professor there since March 1992 until his retirement from Kyoto University in March, 2013. During the last 35 years, he has been mainly engaged in the research of wireless personal communications. His current research interests include highly spectrally efficient wireless transmission techniques and wireless distributed networks. From 1990 to 1991, he was a visiting scholar at WINLAB, Rutgers University, U.S.A. and Carleton University in Ottawa, Canada. He served as a General Co-Chair of IEEE VTC 2012Spring, Yokohama, and a General Chair of the APWCS 2012, Kyoto. He received the IEICE Achievement Award, Ericsson Telecommunication Award, IEICE Best Paper Award and Okawa Prize in 1993, 2007, 2011, and 2013, respectively. 